\title{
Assessing International Commonality in Macroeconomic Uncertainty and Its Effects*
}

\author{
Andrea Carriero \\ Queen Mary, University of London \\ Todd E. Clark \\ Federal Reserve Bank of Cleveland \\ Massimiliano Marcellino \\ Bocconi University, IGIER and CEPR
}

September 2019

\begin{abstract}
This paper uses a large vector autoregression to measure international macroeconomic uncertainty and its effects on major economies. We provide evidence of significant commonality in macroeconomic volatility, with one common factor driving strong comovement across economies and variables. We measure uncertainty and its effects with a large model in which the error volatilities feature a factor structure containing time-varying global components and idiosyncratic components. Global uncertainty contemporaneously affects both the levels and volatilities of the included variables. Our new estimates of international macroeconomic uncertainty indicate that surprise increases in uncertainty reduce output and stock prices, adversely affect labor market conditions, and in some economies lead to an easing of monetary policy.
\end{abstract}

Keywords: Business cycle uncertainty, stochastic volatility, large datasets

J.E.L. Classification: F44, E32, C55, C11

\footnotetext{
*Corresponding author: Todd E. Clark, todd.clark@researchfed.org. We gratefully acknowledge research assistance from John Zito and helpful comments from Efrem Castelnuovo, Alessandro Rebucci, editor Barbara Rossi, and three anonymous referees. The views expressed herein are solely those of the authors and do not necessarily reflect the views of the Federal Reserve Bank of Cleveland or the Federal Reserve System. Carriero gratefully acknowledges support for this work from the Economic and Social Research Council [ES/K010611/1].
} 


\section{Introduction}

Since the seminal analysis of Bloom (2009), a large body of research has examined the measurement of macroeconomic uncertainty and its effects. Bloom (2014) surveys related work up through several years ago. Additional recent contributions include, among others, Baker, Bloom and Davis (2016), Basu and Bundick (2017), Caggiano, Castelnuovo, and Groshenny (2014), Carriero, Clark, and Marcellino (2018, 2019), Gilchrist, Sim, and Zakrajsek (2014), Jurado, Ludvigson, and Ng (2015), Leduc and Liu (2016), and Ludvigson, Ma, and Ng (2019).

Although much of the literature has focused on uncertainty within a single economy, some work has examined common international aspects of uncertainty and its effects. Among studies of economic models, Gourio, Siemer, and Verdelhan (2013) develop an international real business cycle model in which an increase in the probability of disaster leads to a decline in GDP, investment, and employment, with larger effects on the economy that would be more affected by the disaster. After developing economic evidence of international comovement in volatilities (discussed in more detail below, in a separate section on empirical evidence), Mumtaz and Theodoridis (2017) build a two-economy, dynamic stochastic general equilibrium (DSGE) model in which cross-country risk sharing (for consumption smoothing) and trade openness help to drive such comovement of volatilities. Cross, Kam, and Poon (2018) develop a two-economy DSGE model in which level and volatility shocks can have real effects.

Other studies take a comparative international perspective, while not allowing for crosscountry interactions. For example, Fernández-Villaverde, Guerrón-Quintana, Rubio-Ramírez, and Uribe (2011) focus on the effects of changes in the stochastic volatility of the real interest rate in small EMEs. After estimating the volatility process, they insert it in a small open economy DSGE model, and obtain that a increase in the real interest rate volatility leads to a decrease in output, consumption, investment, hours, and debt.

Extending prior empirical work on international aspects of uncertainty (as detailed below), in this paper we use large Bayesian vector autoregressions (BVARs) to measure international macroeconomic uncertainty and its effects on major economies. We do so for two datasets, one consisting of GDP growth for 19 industrialized economies and the other comprised of 67 variables in quarterly data for the U.S., euro area (E.A.), and U.K. We first use basic factor model diagnostics to assess the common factor structure of the stochastic volatilities of BVARs. Then, to estimate global uncertainty and its effects, we turn to our preferred large, heteroskedastic VAR in which the error volatilities evolve over time according to a factor structure, as developed in the U.S.-only analysis of Carriero, Clark, and Marcellino (2018). The volatility of each variable 
in the system reflects time-varying common (global) components and idiosyncratic components. In this model, global uncertainty is allowed to contemporaneously affect the macroeconomies of the included nations - both the levels and volatilities of the included variables. Changes in the common components of the volatilities of the VAR's variables provide contemporaneous, identifying information on uncertainty. Uncertainty and its effects are estimated in a single step within the same model.

Our results point to significant commonality in international macroeconomic volatility, with one common factor - our measure of global uncertainty - accounting for strong comovement across economies and variables in each of our datasets. Our global uncertainty measure is strongly correlated with a comparable measure for the U.S. from Carriero, Clark, and Marcellino (2018) and to a modestly lesser extent with the Jurado, Ludvigson, and Ng (2015) estimate of U.S. macroeconomic uncertainty. This suggests that global macroeconomic uncertainty is closely related to uncertainty in the U.S., which might not seem surprising given the tie of the international economy to the U.S. economy. Our estimate of global macroeconomic uncertainty appears to be more modestly correlated with estimates of financial uncertainty from the literature and the global economic policy uncertainty measure of Davis (2016).

Our results also include impulse response functions for a surprise increase in global macroeconomic uncertainty. According to these estimates, a shock to global uncertainty reduces GDP in most industrialized countries. In the larger set of indicators for the U.S., E.A., and U.K., the surprise increase in uncertainty lowers GDP and many of its components, adversely affects labor market conditions, lowers stock prices, and in some economies leads to an easing of monetary policy. Our identified global uncertainty shock is uncorrelated with other structural (U.S.-based) shocks, such as productivity, fiscal, or monetary shocks. Hence, the responses are capturing a genuine effect from unexpected increases in uncertainty.

Historical decomposition estimates for the 19-country GDP dataset indicate that, while shocks to uncertainty can have noticeable effects on GDP growth in many countries, on balance they are not a primary driver of fluctuations in macroeconomic and financial variables. For example, over the period of the Great Recession and subsequent recovery, shocks to uncertainty made modest contributions to the paths of GDP growth in many countries (e.g., U.S., France, Spain, and Sweden) and small contributions in some countries (e.g., Japan and Norway). In the declines of GDP growth observed in a number of countries in the early 1990s and early 2000s, uncertainty shocks made small contributions in some countries (e.g., U.S., Sweden, and U.K.). Overall, shocks to the VAR's variables played a much larger role than did uncertainty shocks. However, there is a sense in which that is a natural result of considering the VAR shocks jointly as a set 
versus the uncertainty shock by itself; individually, some or many of the VAR shocks would also play small or modest roles.

The paper is structured as follows. Section 2 reviews related empirical literature and explains our contributions. Section 3 describes the data. Section 4 uses basic factor model diagnostics to assess the global factor structure in macroeconomic volatility. Section 5 introduces our preferred large BVAR model for measuring uncertainty and its effects and then presents results. Section

6 describes some robustness checks. Section 7 summarizes our main findings. The supplemental appendix details the estimation algorithm and priors and provides additional results.

\section{Relationship to Prior Work}

To make clear our contributions, in this section we first briefly summarize the most closely related empirical studies of common international aspects of uncertainty and its effects. We then detail key differences in our analysis compared to the most closely related prior work. In broad terms, our work extends the literature by a combination of the use of a large dataset for each economy considered, the inclusion of uncertainty (volatility factors) in the conditional mean of the VAR, and the joint, one-step estimation of uncertainty and its first-moment macroeconomic effects. Our approach also differs from some others in that our uncertainty measure is a common factor in macroeconomic volatilities, whereas in some research uncertainty is measured with the volatility of common factors in the business cycle (i.e., factors in first moments).

In a dataset of 243 variables for 11 industrialized countries, Mumtaz and Theodoridis (2017) apply a factor model with stochastic volatility components common to the world and each country. They find the global component to be an important driver of time-varying volatility. Using GDP growth for 20 countries, Berger, Grabert, and Kempa (2016) estimate a factor model with stochastic volatility components common to the world and specific to each country; in a second step, for each country, they estimate VARs with other variables and uncertainty to assess the effects of uncertainty. Carriere-Swallow and Cespedes (2013) and Gourio, Siemer, and Verdelhan (2013) also use simple, small VAR approaches, measuring uncertainty with the volatility of stock returns. Using 45 variables for G-7 nations, Cuaresma, Huber, and Onorante (2017) apply a VAR with common factors in shocks that have a time-varying variance represented with stochastic volatility. Their estimates yield a common factor that is closely tied to the volatility of global equity prices, and shocks to that factor have significant macroeconomic and financial effects. Cross, Hou, and Poon (2018) use a VAR with common stochastic volatility in mean to estimate the effects of domestic and international uncertainty in three small open economies, 
finding that international uncertainty spillovers have important effects in all countries.

Some other analyses have assessed international comovement in financial uncertainty. Using data on realized stock return volatility and GDP growth in 33 countries, Cesa-Bianchi, Pesaran, and Rebucci (2019) show that return volatility is much more correlated across countries than is GDP growth, that global growth has a sizable contemporaneous impact on financial volatility, and that a common factor accounts for the bulk of the correlation between return volatility and growth. Casarin, Foroni, Marcellino, and Ravazzolo (2018) propose a Bayesian panel model for mixed frequency data, with random effects and parameters changing over time according to a Markov process, to study the effects of macroeconomic and financial uncertainty on a set of 11 macroeconomic variables per country, for a set of countries including the U.S., several European countries, and Japan. In their analysis, macroeconomic uncertainty is measured by the cross-sectional dispersion in survey forecasts of GDP growth, and financial uncertainty is measured by the VIX for the U.S. They find that, for most of the variables, financial uncertainty dominates macroeconomic uncertainty, and the effects of uncertainty differ depending on whether the economy is in a contraction or expansion regime.

Other research has focused on the international transmission of policy uncertainty 1 From a nonlinear (smooth transition) VAR, Caggiano, Castelnuovo, and Figueres (2019) find significant asymmetries, with Canadian (and U.K.) unemployment increasing after a U.S. uncertainty shock in recessions but not expansions. Their interpretation of the results is that higher U.S. policy uncertainty leads to higher uncertainty in Canada and the U.K., and this in turn affects economic activity. Belke and Osowski (2019) compare the transmission of U.S. and E.A. policy uncertainty using a large-scale FAVAR model with data for 18 OECD countries. Their results are broadly in line with those of Caggiano, Castelnuovo, and Figueres (2019), in the sense that the effects of both U.S. and E.A. uncertainty shocks are generally negative on all countries, with stronger effects for the former than for the latter, and with uncertainty shocks originating in one country quickly increasing uncertainty in the other countries.

As to the relationship of our paper to prior studies focused on macroeconomic uncertainty related to time-varying second moments, our model is closely related to that developed in the U.S.-only analysis of Carriero, Clark, and Marcellino (2018). In this paper, we exploit the flexi-

\footnotetext{
${ }^{1}$ Bhattarai, Chatterjee, and Park (2019) examine the transmission of U.S. uncertainty as measured with the VIX to emerging market economies, finding significant effects with some heterogeneity across countries tied to differences in monetary policy responses. Still other studies have examined other global aspects of uncertainty with network-based approaches: Klossner and Sekkel (2014) find evidence of significant spillovers of policy uncertainty from one advanced economy to policy uncertainty in another, and Rossi and Sekhposyan (2017) find spillovers of macroeconomic uncertainty among Euro area countries.
} 
bility of the underlying framework of our earlier work to adjust it to fit our international context. For example, as detailed below, in our two factor implementation, only one (this paper) rather than both factors (earlier paper) enter the conditional mean, and the idiosyncratic components are constant (this paper) rather than time-varying (earlier paper). This paper's primary contribution is empirical, adapting our previous U.S.-only framework to assess the commonality in macroeconomic uncertainty across countries and its effects on major economies.

As a general matter, we believe that our approach and analysis has some possible advantages over some prior work on international uncertainty by allowing and making use of more variables per country. In the existing studies that have assessed the effects of uncertainty on macroeconomic fluctuations across countries, uncertainty has commonly been measured and assessed using a small set of variables for each country. For example, Berger, Grabert, and Kempa (2016) assess the effects of uncertainty using seven variables per country, and Cross, Hou, and Poon (2018) use three variables per economy, pairing one large economy with one small economy. In our larger data set, we have roughly 25 variables for each of the U.S. and E.A. Other work in the uncertainty literature, including Jurado, Ludvigson, and Ng (2015) and Carriero, Clark, and Marcellino (2018), has emphasized some benefits to using relatively large cross sections. In particular, the use of small VAR models to assess the effects of uncertainty can make the results subject to the common omitted variable bias and non-fundamentalness of the errors, and it can assess uncertainty's impacts on only a small number of economic indicators.

More specifically, as regards the relationship of our paper to Mumtaz and Theodoridis (2017) and Cuaresma, Huber, and Onorante (2017), we believe our paper provides a more direct assessment of the macroeconomic effects of uncertainty (as typically formulated in the literature, such as in Jurado, Ludvigson, and Ng 2015). The Mumtaz and Theodoridis (2017) model has common factors in macroeconomic data, with stochastic volatility in the factor innovations driven by common factors in volatilities. In their setup, the common volatility factors, which we would view as aggregate uncertainty, are not in the conditional mean of the factor model (i.e., the levels of the data). They go on to assess the role of the international volatility factors in fluctuations in second moments, but not first moments. We instead focus on the typical issue in the singlecountry uncertainty literature: the effects of volatility factors (uncertainty) on first moments. To that end, we include uncertainty in the conditional mean of the model and conduct impulse response analysis not possible in the Mumtaz and Theodoridis (2017) framework. Put another way, our contribution is to answer a question about effects of uncertainty on business cycle (first moment) fluctuations not addressable with the Mumtaz and Theodoridis (2017) model. If the goal were a narrower one of only estimating global economic uncertainty, it would not be essential 
to include the first moment link of our model. Unreported comparisons to a version of our model without the first moment effects indicate that, while the first moment link clearly affects and informs the estimate of uncertainty, the restricted estimate of global uncertainty is significantly correlated with our baseline estimate.

Cuaresma, Huber, and Onorante (2017) use a VAR coupled with a factor model of the VAR's innovations. Innovations to the factor have stochastic volatility. However, the time-varying variance does not enter the conditional mean of the VAR. Rather, the factor in the VAR's firstmoment errors appears to be treated as the measure of risk and uncertainty. By assumption, this shock to first moments is uncorrelated with innovations to second moments (that is, innovations to the volatility process). In this sense, their results may confound first-moment shocks with second-moment changes. In the broader uncertainty literature (e.g., Jurado, Ludvigson, and Ng 2015), it is more common to define an uncertainty shock by a shock to second moments. Our paper follows this tradition, defining uncertainty as a common factor in forecast error variances that can have first-moment effects (i.e., appears in the VAR's conditional mean).

One other difference between our paper and a number of others in the international literature is that we focus on large economies whereas others focus on small advanced or emerging market economies. As examples, Carriere-Swallow and Cespedes (2013) focus on the effects of uncertainty on emerging market economies, and Cross, Hou, and Poon (2018) concentrate on small advanced economies.

While our paper shares with Berger, Grabert, and Kempa (2016) the focus on effects of shocks to macroeconomic uncertainty as most commonly conceived, we believe our approach offers a couple of advantages. One, as noted above, is that we consider a larger dataset (in part of our results; we also consider a GDP-only dataset, as do they). The other is that our approach assesses uncertainty and its effects with an internally-consistent one-step approach, rather than the two-step approach used in Berger, Grabert, and Kempa (2016) and common in other studies in the uncertainty literature. In the two-step approach, a measure of uncertainty is estimated in a preliminary step and then used as if it were observable data in the subsequent econometric analysis of its impact on macroeconomic variables. However, as described in Carriero, Clark, and Marcellino (2018), with such a two-step approach, it is possible that measurement error in the uncertainty estimate could lead to endogeneity bias in estimates of uncertainty's effects, and the uncertainty around the uncertainty estimate is not easily accounted for in such a setup, since the proxy for uncertainty is treated as data. Moreover, the models used in the first and second steps are somewhat contradictory, with the first step treating second moments as time-varying and the second treating them as constant over time. As our results below show, the two-step approach 
tends to overestimate the precision of impulse response estimates compared to our preferred onestep approach that accounts for uncertainty around the measure of macroeconomic uncertainty. We acknowledge, however, a tradeoff with respect to potential model misspecification: were our preferred model to sufficiently mis-specify the uncertainty process and its link to economic conditions, it might yield estimates of uncertainty and its effects more biased than could be obtained with a two-step approach.

One final matter is the concept of uncertainty. The uncertainty literature features a range of both concepts and measures, described in Bloom (2014). Some studies (e.g., Basu and Bundick 2017 and Caggiano, Castelnuovo, and Groshenny 2014) use stock volatility-based measures, whereas others (e.g., Baker, Bloom, and Davis 2016 and Caggiano, Castelnuovo, and Figueres 2019) rely on measures uncertainty associated with economic policies, based on newspaper coverage. Jurado, Ludvigson, and $\mathrm{Ng}$ (2015) argue for defining and measuring uncertainty with forecast error variances. Rossi, Sekhposyan, and Soupre (2018) provide additional discussion of alternative concepts and measures, including a distinction between ex post and ex ante discussion. The measures of uncertainty we use in this paper are based on the concept of Jurado, Ludvigson, and $\mathrm{Ng}$ (2015), and below we will discuss their correlation with some of the alternatives.

\section{Data}

As indicated above, we rely on two datasets, one consisting of GDP growth rates for a relatively large set of industrialized economies and the other consisting of a larger set of macroeconomic variables for three large economies. Although the first dataset is similar to others in the literature and helps to establish an international factor structure to uncertainty, our greater interest is in the second dataset because it includes relatively large variable sets for each economy.

More specifically, for the GDP growth analysis, we use quarterly data on GDP in 19 industrialized economies, obtained from the OECD's online database (OECD 2017); Table 1 provides the country list. This country set is the same as that in Berger, Grabert, and Kempa (2016), except that we omit New Zealand due to data missing early in our sample. Although we could include more countries in this sample, we don't do so partly out of consideration of model stability. Instabilities seem likely to increase with larger cross-sections of countries, due to differences over time or across countries in economic development.

For the analysis of a wider set of macroeconomic indicators across industrialized economies, long time series on large variable sets are difficult to find. Accordingly, we focus on a few major economies for which relatively large sets of long time series are available: the U.S., euro area, 
and U.K. For the U.S. and E.A., we obtain quarterly data on major macroeconomic indicators from the files of Jarocinski and Mackowiak (2017). After omitting their series with missing data and a few others (for various reasons, including overlap with other series), we use 51 variables from their dataset, 26 for the U.S. and 25 for the E.A.2 For the U.K., we obtained comparable data on 16 variables from Haver Analytics. Table 2 lists the variables.

This specification reflects some choice as to what constitutes a "macroeconomic" variable for the purpose of measuring uncertainty and its effects. Common large datasets used in factor model or FAVAR analysis include a number of indicators - of stock prices, interest rates, and exchange rates - that may be considered financial indicators. In our model specification, the variables that might be considered more financial than macroeconomic are the bond yields and aggregate stock returns. [Short-term interest rates - the instruments of monetary policy seem to be appropriately treated as macroeconomic variables.] In our baseline specification, we follow the precedents of Jurado, Ludvigson, and Ng (2015) and Ludvigson, Ma, and Ng (2019) in including selected bond yields and aggregate stock returns in a macroeconomic model. We have verified that the main results presented below are robust to dropping the bond yields and stock returns from the estimation.

In the applications using both datasets, we follow common practice in the factor model literature and transform each data series for stationarity as needed. With the GDP dataset, we use quarter-on-quarter growth rates computed as log differences. With the 3-economy dataset, we use similar transformations as appropriate; Appendix Table 1 lists any transformations used to achieve stationarity of these data. We also follow common practice in the factor model literature as well as as Jurado, Ludvigson, and Ng (2015) and Carriero, Clark, and Marcellino (2018) by standardizing the data (demean and divide by the simple standard deviation) before estimating the model. Note that, although the model is estimated with standardized data, for comparability to previous studies the impulse responses are scaled and transformed back to the units typical in the literature ${ }^{3}$ The fact that the models are estimated using some variables differenced for stationarity (e.g., GDP, consumption, and investment) implies that, for some of these variables, the long-run effects of uncertainty shocks on their levels will not entirely die out.

\footnotetext{
${ }^{2}$ More specifically, of the variables used by Jarocinski and Mackowiak (2017), we omit: series missing data early in our sample (stock market volatility, bond spreads, and house prices); other private credit-related measures (loans to NFCs, lending rate to NFCs, and mortgage interest rate); narrower measures of the money supply (M1 for the U.S. and M1 and M2 for the E.A.); and some additional variables that rank poorly in their causal priority results (exchange rates and government debt).

${ }^{3}$ We do so by using the model estimates to: (1) obtain impulse responses in standardized, sometimes (i.e., for some variables) differenced data; (2) multiply the impulse responses for each variable by the standard deviations used in standardizing the data before model estimation; and (3) accumulate the impulse responses of step (2) as appropriate to get back impulse responses in levels or log levels.
} 
The time samples used in estimation reflect data availability, any transformations used for stationarity, and considerations of sample stability. In the baseline estimates with the 19-country GDP dataset, the sample is 1985:Q1-2016:Q3. In this case, although the available data permit a longer sample, we use a 1985 start to reduce the chances that changes in policies or other structural forces of economies more likely over a longer sample induce parameter instabilities (section 6 of the supplemental appendix summarizes a check of robustness in an estimation sample starting in 1960). In estimates with the 3-economy macroeconomic dataset, the sample is 1985:Q4-2013:Q3, reflecting the span of the Jarocinski-Mackowiak dataset.

\section{Commonality in International Uncertainty}

To assess the global factor structure of macroeconomic uncertainty, we apply to estimates of the stochastic volatilities of BVARs the basic factor model diagnostics surveyed and used in applications by Stock and Watson (2016). The volatility estimates are posterior medians of log stochastic volatilities obtained from conventional BVARs with stochastic volatility (denoted BVAR-SV; due to space restrictions, the by-now familiar model is provided in the appendix's section 3.2 and not the text) 1 We consider the marginal $R^{2}$ of volatility factors estimated by principal components and the Ahn and Horenstein (2013) eigenvalue ratio.

For GDP growth in 19 countries, the measures of factor structure suggest one strong factor in the international volatility of the business cycle. The first factor accounts for an average of about 79 percent of the variation in log volatilities (see Appendix Table 2 for detailed estimates). The second and third factors account for about 11 and 6 percent, respectively. The Ahn-Horenstein ratio peaks at one factor with a value of 7.4, compared to 1.7 and 3.0 for the second and third factors, respectively. As reported in Table 1, the factor loadings associated with the principal components are fairly tightly clustered around 1, with a minimum of 0.751 for Denmark and a maximum of 1.114 for Sweden. In this sense the common volatility factor puts comparable weight on each country's volatility.

For the larger set of macroeconomic indicators for the U.S., E.A, and U.K., we use volatility estimates from BVAR-SV models fit for each economy to assess the commonality in volatility Figure 1 compares volatility estimates across these three economies for a subset of major macroe-

\footnotetext{
${ }^{4}$ In this model, the VAR's innovation vector takes the form $v_{t}=A^{-1} \Lambda_{t}^{0.5} \epsilon_{t}$, with the diagonal matrix $\Lambda_{t}$ containing the stochastic volatilities, and the reduced-form error variance matrix is $\Sigma_{t}=A^{-1} \Lambda_{t} A^{-1 \prime}$. Our reported results are based on 5,000 draws, obtained by sampling a total of 30,000 draws, discarding the first 5,000 , and retaining every 5 th draw of the post-burn sample.

${ }^{5}$ We estimate the model separately for each country rather than as one single system to avoid an unduly informative proper prior on the log volatility innovation variance matrix $\Phi$.
} 
conomic indicators (we use a subset to limit the number of charts). In this comparison, volatility is reported in the way common in the literature, as the (posterior median of the) standard deviation of the reduced-form innovation in the BVAR. Qualitatively, these estimates suggest considerable commonality within and across countries. As the chart indicates, for a given country, there is significant comovement across variables. For example, for the U.S., most variables display a rise in volatility around the recessions of the early 1990s, 2001, and 2007-2009. For the E.A., most variables display sizable increases in uncertainty in the early and mid-1990s and again with the Great Recession. In addition, there appears to be significant comovement across economies, somewhat more so for volatility in the U.S. and E.A. than in the case of the U.K.

In the 3-economy macroeconomic dataset, a first factor accounts for an average of about 42 percent of the variation in log volatilities (see Appendix Table 2 for details). The role of the first factor in volatility is much stronger in this dataset (and in the 19-country GDP dataset) than in the monthly U.S. data of Jurado, Ludvigson, and $\mathrm{Ng}$ (2015). For most variables, the estimated loadings on this factor reported in Table 2 are clustered around a value of 1 . For example, the loadings on GDP growth are 1.330 for the U.S., 1.288 for the E.A., and 1.188 for the U.K. In this sense the common volatility factor puts comparable (but not equal) weight on the volatility of most variables in the model. Overall, the patterns in the estimated loadings appear consistent with an interpretation in which the first factor is capturing a common component in macroeconomic volatilities, with most loadings clustered around values of 1 , most prominently for the U.S. variables, almost as clearly for the E.A., and with modestly more dispersion in loadings on the U.K. variables. A second factor accounts for about 26 percent of the variation in international macroeconomic volatility. Together, two factors account for more than 68 percent of the variation in volatility across indicators and countries. Subsequent factors account for significantly smaller marginal shares of variation. The Ahn-Horenstein ratio peaks at two factors. Together, the $R^{2}$ and Ahn-Horenstein estimates suggest two factors in this larger dataset.

The detected substantial commonality in volatilities is broadly in line with the empirical evidence of Mumtaz and Theodoridis (2017), as well as the prediction of their DSGE model, in which commonality is driven by cross-country risk sharing, globalization, and trade openness.

\section{Measuring the Impact of Uncertainty}

Having established evidence of common factors in international macroeconomic volatilities, we now turn to assessing the effects of global uncertainty on macroeconomic fluctuations. This section begins by detailing the Bayesian VAR with a generalized factor structure - henceforth 
referred to as a BVAR-GFSV model — we use for that purpose, first for a one-factor model applied to the 19-country GDP dataset and then for a two-factor specification applied to the 3-economy macroeconomic dataset ${ }^{6}$ We then present results for the uncertainty estimates and effects of shocks to uncertainty.

\subsection{One-Factor BVAR-GFSV Model}

With the evidence in the previous section pointing to one factor in the 19-country GDP dataset, we rely on a one-factor model in our baseline results for the dataset.

Let $y_{t}$ denote the $n \times 1$ vector of variables of interest - covering multiple countries. The $n \times 1$ vector of reduced-form shocks to these variables is:

$$
v_{t}=A^{-1} \Lambda_{t}^{0.5} \epsilon_{t}, \epsilon_{t} \sim \operatorname{iid} N(0, I)
$$

where $A$ is an $n \times n$ lower triangular matrix with ones on the main diagonal, and $\Lambda_{t}$ is a diagonal matrix of volatilities, $\lambda_{i, t}, i=1, \ldots, n$. For each variable $i$, its log-volatility follows a linear factor model with a common uncertainty factor $\ln m_{t}$ that follows an $\operatorname{AR}\left(p_{m}\right)$ process augmented to include $y_{t-1}$ and an idiosyncratic component $\ln h_{i, t}$ that follows an $\mathrm{AR}(1)$ process:

$$
\begin{aligned}
\ln \lambda_{i, t} & =\beta_{m, i} \ln m_{t}+\ln h_{i, t}, i=1, \ldots, n \\
\ln m_{t} & =\sum_{i=1}^{p_{m}} \delta_{m, i} \ln m_{t-i}+\delta_{m, y}^{\prime} y_{t-1}+u_{m, t}, u_{m, t} \sim i i d N\left(0, \phi_{m}\right) \\
\ln h_{i, t} & =\gamma_{i, 0}+\gamma_{i, 1} \ln h_{i, t-1}+e_{i, t}, i=1, \ldots, n .
\end{aligned}
$$

The volatility factor $m_{t}$ is our measure of (unobservable) global macroeconomic uncertainty. The idiosyncratic component $h_{i, t}$ captures time variation in a country's GDP volatility unique to that country. The uncertainty shock $u_{m, t}$ is independent of the conditional errors $\epsilon_{t}$ and the vector of volatility innovations $\nu_{t}=\left(e_{1, t}, \ldots, e_{n, t}\right)^{\prime}$, which is jointly distributed as iid $N\left(0, \Phi_{\nu}\right)$ with elements independent among themselves, so that $\Phi_{\nu}=\operatorname{diag}\left(\phi_{1}, \ldots, \phi_{n}\right)$. For identification, we follow common practice in the dynamic factor model literature and assume $\ln m_{t}$ to have a zero unconditional mean, fix the variance $\phi_{m}$ at 0.03 , and use a simple accept-reject step to restrict the first variable's (U.S. GDP growth) loading to be positive.

The global uncertainty measure $m_{t}$ can also affect the levels of the macroeconomic variables

\footnotetext{
${ }^{6}$ In light of space restrictions, we refer the reader to the supplemental appendix's sections 2 and 3.1 and Carriero, Clark, and Marcellino 2018 for details of the Gibbs sampler and priors used to estimate the model. Our reported results are based on 5,000 draws, obtained by sampling a total of 30,000 draws, discarding the first 5,000 , and retaining every 5 th draw of the post-burn sample.
} 
contained in $y_{t}$, contemporaneously and with lags. In particular, $y_{t}$ is assumed to follow:

$$
y_{t}=\sum_{i=1}^{p} \Pi_{i} y_{t-i}+\sum_{i=0}^{p_{m}} \Pi_{m, i} \ln m_{t-i}+v_{t},
$$

where $p$ denotes the number of $y_{t}$ lags in the VAR, $p_{m}$ denotes the number of $\ln m_{t}$ lags in the conditional mean of the VAR (for computational convenience, set to the lag order of the factor process), $\Pi_{i}$ is an $n \times n$ matrix, $i=1, \ldots, p$, and $\Pi_{m, i}$ is an $n \times 1$ vector of coefficients, $i=0, \ldots, p_{m}$.

This model allows the international business cycle to respond to movements in global uncertainty, both through the conditional variances (contemporaneously, via movements in $v_{t}$ ) and through the conditional means (contemporaneously and with lag), via the coefficients collected in $\Pi_{m, i}, i=0, \ldots, p_{m}$. In our implementation, we set the model's lag orders at $p=2$ and $p_{m}$ $=2$. Note that $y_{t}$ cannot contemporaneously affect uncertainty, which in this sense is treated as exogenous. (However, it is not entirely exogenous: The model allows uncertainty to respond with a lag to macroeconomic conditions. We return to endogeneity questions after presenting the two-factor model next.)

\subsection{Two-Factor BVAR-GFSV Model}

With Section 4's principal component-based analysis of volatilities obtained from BVAR-SV estimates pointing to two factors in the 3-economy macroeconomic dataset, we also consider specifications with two common volatility components. The natural starting point would be the model described above extended to include a second factor in both the volatility process and the VAR's conditional mean. In unreported estimates, we considered such a model. The estimate of the first factor in this unrestricted two-factor specification was very similar to the estimate obtained from a one-factor specification and strongly correlated with the first principal component of BVAR-SV volatilities. The estimated second factor seemed to capture a modest low-frequency decline in volatility from the first half of the sample to the second half, with generally insignificant effects on the levels of the variables. However, these results from an unrestricted two-factor specification appear to suffer problems with the convergence of the Markov Chain Monte Carlo (MCMC) sampler with this dataset (although not with other datasets).

From this analysis, we conclude that although there are two volatility or uncertainty factors in the 3-economy macroeconomic dataset, only one bears on the levels of macroeconomic variables. As we describe in more detail in the robustness section below, we obtained a qualitatively similar

result with an alternative simple approach of adding to the macroeconomic BVAR the principal components of the BVAR-SV volatilities used in this section. 
Accordingly, for the 3-economy macroeconomic dataset, our baseline results use a two-factor model with some restrictions. In particular, the model features two common factors in volatilities but includes only one of the factors in the conditional mean of the VAR and affecting the levels of the included variables. In addition, reflecting other evidence, the idiosyncratic component of volatility is simply a constant. With the larger set of indicators for the U.S., E.A., and U.K. in our sample of quarterly data starting in 1985, unreported estimates of a version of the model with an $\mathrm{AR}(1)$ process for the idiosyncratic component of volatility - a specification that yields results very similar to those we report - display very little time variation in the idiosyncratic components. For the 3-economy macroeconomic dataset, our model estimates attribute the vast majority of time variation in volatility to the common component $m_{t}$.

With these restrictions, the model applied to the 3-economy macroeconomic dataset takes the following form, including two international uncertainty factors $m_{t}$ and $f_{t}$ :

$$
\begin{aligned}
y_{t} & =\sum_{i=1}^{p} \Pi_{i} y_{t-i}+\sum_{i=0}^{p_{m}} \Pi_{m, i} \ln m_{t-i}+v_{t} \\
v_{t} & =A^{-1} \Lambda_{t}^{0.5} \epsilon_{t}, \epsilon_{t} \sim i i d N(0, I) \\
\ln \lambda_{i, t} & =\beta_{m, i} \ln m_{t}+\beta_{f, i} \ln f_{t}+\ln h_{i}, i=1, \ldots, n \\
\ln m_{t} & =\sum_{i=1}^{p_{m}} \delta_{m, i} \ln m_{t-i}+\delta_{m, y}^{\prime} y_{t-1}+u_{m, t}, u_{m, t} \sim i i d N\left(0, \phi_{m}\right) \\
\ln f_{t} & =\sum_{i=1}^{p_{f}} \delta_{f, i} \ln f_{t-i}+\delta_{f, y}^{\prime} y_{t-1}+u_{f, t}, u_{f, t} \sim i i d N\left(0, \phi_{f}\right) .
\end{aligned}
$$

In this case, the log-volatility of each variable $i$ follows a linear factor model with common unobservable uncertainty factors $\ln m_{t}$ and $\ln f_{t}$, which follow independent AR processes augmented to include $y_{t-1}$, and a constant idiosyncratic component $\ln h_{i}$. The volatility factors $m_{t}$ and $f_{t}$ are measures of (unobservable) global macroeconomic uncertainty. However, only the first global uncertainty measure, $m_{t}$, enters the conditional mean of the VAR and affects the levels of the macroeconomic variables contained in $y_{t}$, contemporaneously and with lags. The time-invariant idiosyncratic component captures differences in the average level of volatility across economies.

To spell out the notation, which follows that used in the one-factor model above, $A$ is an $n \times n$ lower triangular matrix with ones on the main diagonal; $\Lambda_{t}$ is a diagonal matrix of volatilities, $\lambda_{i, t}, i=1, \ldots, n ; p$ denotes the number of $y_{t}$ lags in the VAR; $p_{m}$ denotes the number of $\ln m_{t}$ lags in the conditional mean of the VAR; $\Pi_{i}$ is an $n \times n$ matrix, $i=1, \ldots, p$; and $\Pi_{m, i}$ is an $n \times 1$ vector of coefficients, $i=0, \ldots, p_{m}$. The uncertainty shocks $u_{m, t}$ and $u_{f, t}$ are independent of each other and independent of the conditional errors $\epsilon_{t}$. For identification, we assume that 
$\ln m_{t}$ and $\ln f_{t}$ have zero unconditional means, fix their variances $\phi_{m}$ and $\phi_{f}$ at 0.03 , and use a simple accept-reject step to restrict the first factor's loading on U.S. GDP growth and the second factor's loading on E.A. GDP growth to be positive. In our implementation, we set the model's lag orders at $p=2, p_{m}=2$, and $p_{f}=2$.

In results omitted in the interest of brevity, we have obtained similar findings with some other modifications of this two-factor model. In one robustness check, we extended the factor process of equations (9) and (10) to allow VAR dynamics rather than AR dynamics in the factors and allow a non-zero correlation between the innovations to the factors. We also considered specifications with up to three factors in volatilities linked to various combinations of the three economies. These estimates continued to point to two common factors, with only one having conditional mean effects - similar to those reported - and with factors similar to those reported. Both the simple factor evidence of section 4 and various specifications of the BVAR-GFSV model point to the data being consistent with just two uncertainty factors.

\subsubsection{Rationale for some choices in the baseline model}

Before moving on to empirical results, we take up five specific questions related to our choice of a baseline model. First, in light of the specification of Mumtaz and Theodoridis (2017) that features global, regional, and country factors in volatility, why not add such factors to our model? Basically, the data we use - different in coverage from the data of Mumtaz and Theodoridis (2017) that do support more factors - don't seem to warrant it. As the simple factor analysis of section 4 indicates, and as we will discuss with results for our BVAR-GFSV specifications, the evidence points to two factors and not more. For example, once we allow for two factors in the three-economy dataset, there isn't any meaningful time variation in idiosyncratic components (so, as noted above, we modify the model of our previous paper to treat them as constant).

Second, why specify a VAR structure for the levels of data and a factor model in the volatilities of their innovations? Admittedly, along various dimensions, VARs and factor models can both provide effective representations of the data. For variable sets of the size we consider, our estimation method can accommodate a VAR in the levels of the data, and we prefer a VAR over a factor model in order to impose fewer restrictions on dynamics. In the case of the volatilities and uncertainty, given our treatment of macroeconomic uncertainty as aggregate or common uncertainty, it is more natural to measure it as a common factor in volatilities (as others in the literature, such as Jurado, Ludvigson, and Ng 2015, have done). Moreover, the empirical evidence of factor structure is somewhat stronger in volatilities than in the levels of the data. For example, in the 19-country GDP dataset, the first principal component accounts for 30 percent 
of variation in GDP growth rates but 79 percent of variation in GDP volatility (as measured in section 4's BVAR-SV results).

Third, why not include idiosyncratic volatility terms in the VAR's conditional mean? One reason is that our analysis (following most of the literature, such as Jurado, Ludvigson, and Ng 2015) focuses on common, aggregate uncertainty. Another, noted above, is that once we allow for two factors, no meaningful variation in idiosyncratic components remains.

Fourth, is the relationship between economic conditions and uncertainty allowed in the model important? Let's start with the response of uncertainty to economic conditions as captured by the coefficient vectors $\delta_{m, y}$ and $\delta_{f, y}$ of the uncertainty factor processes of equations (9) and (10). In additional estimates we produced for 3-economy macroeconomic dataset, when the model is restricted to make $\delta_{m, y}$ and $\delta_{f, y}$ equal 0 , the estimates of uncertainty are considerably smoother than in the paper's baseline, the responses of the E.A. and U.K. variables to the uncertainty shock are attenuated, and those of the policy interest rates switch sign. As to the response of economic conditions to uncertainty, captured by the $\Pi_{m, i}$ coefficients in the VAR's conditional mean (equation (6)), these estimates are generally statistically significantly different from zero, in line with the significant impulse responses we will report in the next section. Although the estimates of uncertainty from the restricted model (here, too, we conducted the check with the 3-economy model) are qualitatively similar to those in the unrestricted model of the paper's baseline, the restricted estimate of macroeconomic uncertainty shows less of a rise around the U.S. recessions of the late 1990s and 2001 than does the baseline estimate. Putting these results together, along both of these dimensions of the model's parameterizations, there appears to be substantial evidence of feedback effects between economic conditions and uncertainty.

Finally, why not allow uncertainty to react contemporaneously to economic conditions? As noted above, the BVAR-GFSV model allows macroeconomic uncertainty to respond to economic conditions, but with a lag ( $y_{t-1}$ enters the factor processes (9) and (10)). In larger models that have some advantages for the measurement of uncertainty in internally consistent one-step approaches, there does not exist an approach that allows contemporaneous feedback between uncertainty and economic conditions. Carriero, Clark, and Marcellino (2019) and Ludvigson, Ma, and Ng (2019) develop approaches that do allow such feedback, but in smaller models that take the uncertainty measure as given and are not readily extended to large datasets such as the one we use. Importantly, for the U.S., Carriero, Clark, and Marcellino (2019), find little evidence of contemporaneous effects of economic conditions on macroeconomic uncertainty; their results provide support for a specification like this paper's in which the economy responds quickly to macroeconomic uncertainty and uncertainty responds to economic conditions with a delay. 


\subsection{BVAR-GFSV Estimates of Uncertainty}

Although the BVAR-GFSV estimates of uncertainty reflect influence from the first moments of macroeconomic data, the estimates are also directly related to the loadings on the common factor in volatility. These loadings (for the 3-economy macroeconomic dataset, we report only the first factor's loadings for brevity) are reported in the last columns of Tables 1 and 2. In the case of the 19-country GDP dataset, the loadings are broadly centered around 1, with a minimum of 0.396 for Sweden and maximum of 1.634 for Germany. In this respect, the loadings estimated from the BVAR-GFSV model are similar to those estimated by principal components applied to $\log$ volatilities of the BVAR-SV model. In the case of the 3-economy macroeconomic dataset, most of the variables have sizable loadings on the volatility factor (keeping in mind that the scale of the loadings reflects the normalization imposed by fixing the innovation variance for identification). Across variables, the average of the loading estimates (posterior means) is 0.75 , with a range of 0.12 to 1.50 ; more than $3 / 4$ of the loadings are above 0.5 .

Figure 2 displays the posterior distribution of the measures of uncertainty obtained from the BVAR-GFSV specification, along with corresponding measures obtained from the first principal component of the log volatilities from the BVAR-SV models. The top panel provides estimates for the 19-country GDP dataset, and the bottom panel reports estimates for the 3-economy macroeconomic dataset. In reporting the BVAR-GFSV estimates, we define uncertainty as the

square root of the common volatility factor $\left(\sqrt{m_{t}}\right)$, corresponding to a standard deviation. Figure 2 also reports the 15\%-85\% credible set bands around our estimated measure of uncertainty, which is correctly considered a random variable in our approach. In the case of the first principal component of BVAR-SV log volatilities, for scale comparability we exponentiate the principal component and then compute (and plot) its square root.

As indicated in Figure 2, the uncertainty factors show significant increases around some of the political and economic events that Bloom (2009) highlights as periods of uncertainty, including the first Gulf war, 9/11, the Enron scandal, the second Gulf war, and the recent financial crisis period. In some cases, increases in uncertainty around such events seem to be defined somewhat more clearly in our larger variable set (bottom panel) than in the GDPonly dataset for 19 countries. But in both cases, the credible sets around the BVAR-GFSV estimates indicate that the uncertainty around uncertainty estimates is sizable. Although we believe it to be important to take account of such uncertainty around uncertainty measures, the estimates obtained with our BVAR-GFSV model are significantly correlated with those obtained from the principal component of the BVAR-SV volatility estimates, more so in the 3-economy 
macroeconomic dataset (correlation of 0.800) than in the 19-country GDP dataset (correlation of 0.641). In results omitted in the interest of brevity, with the larger variable set we obtained similar estimates of common factor volatility (and reduced-form volatilities of the model's variables) in a version of the model extended to treat the idiosyncratic components as time-varying. As noted above, in the 3-economy macroeconomic dataset, essentially all of the time variation in volatilities appears to be due to common international components and not to components operating at a country or variable level.

Figure 3 compares our uncertainty estimates to each other and to other estimates in the literature, including CCM macro and financial uncertainty from Carriero, Clark, and Marcellino (2018); JLN macro and financial uncertainty from Jurado, Ludvigson, and Ng (2015); global economic policy uncertainty (EPU) from Davis (2016); common uncertainty from Mumtaz and Theodoridis (2017); and common uncertainty from Berger, Grabert, and Kempa (2016) 7 As indicated in the top left panel, even though our 3-economy macroeconomic and 19-economy GDP datasets differ significantly in composition, estimates of uncertainty obtained with our BVARGFSV model are quite similar, with a correlation of 0.794 . The estimate from our 3-economy dataset is also significantly correlated with the estimate of U.S. macroeconomic uncertainty from Carriero, Clark, and Marcellino (2018) and to a slightly lesser extent with the Jurado, Ludvigson, and Ng (2015) estimate of U.S. macroeconomic uncertainty. This suggests that global macroeconomic uncertainty is closely related to uncertainty in the U.S., which might not seem surprising given the tie of the international economy to the U.S. economy. On the other hand, we have noted that most variables have significant loadings on the international uncertainty factors. So by this very simple measure, the uncertainty we capture is global and not specific to the U.S.

To shed further light on the relationship between U.S. and global uncertainty, we have conducted some additional checks. Specifically, we have compared the 3-economy factor estimate of macroeconomic uncertainty $m_{t}$ from the paper's baseline to country-specific estimates. We obtained the country-specific measures by estimating the one-factor BVAR-GFSV model separately for the U.S., E.A., and U.K. According to these estimates, each of the country-specific factors is significantly correlated with our estimated international factor, with correlations ranging from 0.6 for the U.K. to 0.8 for the U.S. and E.A. We then regress the baseline estimate of $m_{t}$ on various combinations of the country-specific estimates. In a regression of our baseline estimate on all three economy-specific uncertainty measures, the null that the E.A. and U.K. country-specific factors have zero coefficients is overwhelmingly rejected. These regression results indicate that

\footnotetext{
${ }^{7}$ The underlying CCM and JLN estimates are obtained with monthly data and pertain to a one-month ahead horizon. We use quarterly averages of these estimates.
} 
our international factor is not just a U.S. factor.

Our estimate of global macroeconomic uncertainty appears to be modestly correlated with estimates of financial uncertainty from the literature and the global economic policy uncertainty measure of Davis (2016). Our estimate of global macroeconomic uncertainty is also only modestly correlated with the uncertainty measures of Berger, Grabert, and Kempa (2016) and Mumtaz and Theodoridis (2017), both of which display relatively sharp spikes with the Great Recession. Although the number of differences across specifications makes it difficult to identify which factor might account for the differences in uncertainty estimates, one probably important difference is that our uncertainty measure is a common factor in macroeconomic volatilities, whereas in these papers uncertainty is the volatility of common factors in the business cycle.

A related important empirical issue is whether our estimated uncertainty shocks represent an original source of business cycle fluctuations or are simply correlated with traditional macroeconomic shocks. To check, we have computed the correlations of our estimated global macroeconomic uncertainty shocks with some well-known and available macro shocks for the U.S., drawing on comparable exercises in Stock and Watson (2012), Caldara, et al. (2016), and Carriero, Clark, and Marcellino (2018). These estimates, detailed in (supplemental) Appendix Table 3 in the interest of brevity, indicate that our international uncertainty shocks are not very correlated with "known" macroeconomic shocks in the U.S. At least in this sense, to the extent shocks in the U.S. bear on the international business cycle, our estimated uncertainty shocks seem to truly represent a second-order "variance" phenomenon, rather than a first-order "level" shock. While it would be interesting to also assess the correlation of our uncertainty shocks with macroeconomic shocks for other countries or the global economy, we are not aware of standard sources of shocks like those that exist for U.S. data.

\subsection{Measuring the Impact of Uncertainty: Impulse Response Esti- mates and Historical Decompositions from BVAR-GFSV Model}

Figures 4 and 5 provide the BVAR-GFSV estimates of impulse response functions for a shock to international macroeconomic uncertainty. Starting with the 19-country results in Figure 4, an international shock to macroeconomic uncertainty slowly dies out over several quarters. The rise in uncertainty induces statistically significant, persistent declines in GDP in most of the countries, in line with the association found in Ramey and Ramey (1995) ${ }^{8}$ For example,

\footnotetext{
${ }^{8}$ Written with the idea that volatility corresponds to uncertainty, Ramey and Ramey (1995) use simple comparisons of cross-country averages and panel regressions to show that countries with higher volatility have lower growth, in both an OECD sample and a large sample of countries. Adding common control variables strengthens the estimated negative relationship.
} 
after several quarters, GDP falls about 0.4 percentage point in countries including the U.S., Canada, France, the Netherlands, and the U.K. In general, the magnitudes of the declines are comparable across most countries, although a little less severe in some (e.g., Australia) and more severe in others (e.g., Finland and Sweden). Castelnuovo and Tran (2017) obtain a similar finding of larger uncertainty effects in some countries relative to others. Possible reasons could relate to recessions or the zero lower bound (ZLB) constraint on monetary policy: In some research, uncertainty shocks have larger effects during recessions (e.g., Caggiano, Castelnuovo, Groshenny 2014 and Caggiano, Castelnuovo, and Figueres 2019) or in the presence of the ZLB (e.g., Caggiano, Castelnuovo, and Pellegrino 2017), and Australia faced neither a recession nor the ZLB in the 2007-2009 period.

For space savings and readability, Figure 5 covers a subset of variables in providing impulse response estimates for the 3-economy macroeconomic dataset, and it reports posterior medians and 70 percent credible sets for the U.S. responses but just posterior medians for the E.A. and U.K. (Appendix Figure 1 provides complete estimates.) In the estimates for this dataset, it is also the case that an international shock to macroeconomic uncertainty (to the factor $\ln m_{t}$ in the VAR's conditional mean) gradually dies out over a few quarters. For the U.S., E.A., and U.K, the heightened international uncertainty reduces GDP and components including investment, exports, and imports. In all three economies, employment (see Appendix Figure 1) falls and unemployment rises, and some other measures of economic activity, including confidence or sentiment indicators and capacity utilization, also fall. The shock does not have any consistently significant and negative effects on producer or consumer prices, although there are some effects, such as in the case of the fall in producer prices in the E.A. Although stock prices fall in all three economies, the policy rate falls in the U.S. but is little changed in the E.A. or U.K. In some cases - e.g., for GDP - as measured by posterior medians the response of a given variable for the U.S. is larger than the responses for the E.A. and U.K. But this does not apply to all variables - e.g., exports and stock prices.

In general qualitative terms, these results line up with those obtained with monthly data for the U.S. in Carriero, Clark, and Marcellino (2018), with the exception of stock prices, which in our previous paper were essentially unchanged in response to a macro uncertainty shock but fell in response to a shock to financial uncertainty. For the sake of conciseness, we refer to Carriero, Clark, and Marcellino (2018) for references to economic models that can explain response patterns similar to these. Our empirical results are also in line with those of most of the studies reviewed in Section 2 and based on different methodologies, for example, Fernandez-Villaverde, et al. (2011), Berger, Grabert, and Kempa (2016), or Casarin, et al. (2018). That said, as far as we 
are aware, more research is needed to better understand how changes in economic uncertainty affect one major economy more than another.

Although these impulse responses show that shocks to uncertainty have significant effects, they cannot provide an assessment of the broader cyclical importance of global macroeconomic uncertainty shocks. For that broader assessment, we estimate historical decompositions. In a standard linear model, a historical decomposition of the total $s$-step-ahead prediction error variance of $y_{t+s}$ can be easily obtained by constructing a baseline path (forecast) without shocks, and then constructing the contribution of shocks. With linearity, the sums of the shock contributions and the baseline path equal the data. In our case, the usual decomposition cannot be directly applied because of interactions between $\Lambda_{t+s}$ and $\epsilon_{t+s}$ : Shocks to log uncertainty affect the forecast errors through $\Lambda_{t+s} \epsilon_{t+s}$, and, over time, shocks $\epsilon_{t+s}$ affect $\Lambda_{t+s}$ through the response of uncertainty to lagged y. However, as developed in Carriero, Clark, and Marcellino (2018), it is possible to decompose the total contribution of the shocks into three parts: (i) the direct contributions of the uncertainty shocks $u_{t+s}$ to the evolution of $y$; (ii) the direct contributions of the VAR "structural" shocks $\epsilon_{t+s}$ to the path of $y$, taking account of movements in $\Sigma_{t+s}$ that arise as uncertainty responds to $y$ but abstracting from movements in $\Sigma_{t+s}$ due to uncertainty shocks; and (iii) the interaction between shocks to uncertainty and the structural shocks $\epsilon_{t+s}$. The supplemental appendix's section 4 details the basis of the decomposition.

Figures 6 (19-country GDP dataset) and 7 (3-economy macroeconomic dataset) show the standardized data series, a baseline path corresponding to the unconditional forecast, the direct contributions of shocks to macroeconomic uncertainty, and the direct contributions of the VAR's shocks. The reported estimates are posterior medians of decompositions computed for each draw from the posterior. To save space, the charts provide results for a subset of selected variables. Finally, the decomposition results start in 1987:Q1 for the 19-country GDP dataset and, for better readability, 1998:Q1 for the 3-economy macroeconomic dataset.

As indicated in Figure 6's decomposition estimates for the 19-country GDP dataset, while shocks to uncertainty can have noticeable effects on GDP growth in many countries, on balance they are not a primary driver of fluctuations in growth. For example, over the period of the Great Recession and subsequent recovery, shocks to uncertainty made modest contributions to the paths of GDP growth in many (e.g., U.S., France, Spain, and Sweden) and small contributions in some countries (e.g., Japan and Norway). In the declines of GDP growth observed in a number of countries in the early 1990s and early 2000s, uncertainty shocks made small contributions in some countries (e.g., U.S., Sweden, and U.K.). Overall, shocks to the VAR's variables played a much larger role than did uncertainty shocks. However, there is a sense in which that is a natural 
result of considering the VAR shocks jointly as a set versus the uncertainty shock; individually, some or many of the VAR shocks would also play small or modest roles.

Figure 7's decomposition estimates for the 3-economy macroeconomic dataset paint a broadly similar picture. For example, around the Great Recession (2007-2009 for the U.S.), shocks to macroeconomic uncertainty (the first factor $\ln m_{t}$ ) contribute somewhat to fluctuations in economic activity, including in GDP, business investment, and housing investment, but not much to inflation or stock prices. Similar patterns are evident in the decline in GDP growth observed in the early 2000s. With this dataset, too, the effects of uncertainty shocks are generally dominated by the contributions of the VAR's shocks. Carriero, Clark, and Marcellino (2018) obtain a broadly similar result, as does Benati (2016) with a different approach.

The responses of GDP growth for the U.S., U.K., and some European countries obtained with the GDP-only dataset are larger and more persistent than those resulting from the 3-country multi-variable dataset. Similarly, the contributions of uncertainty shocks to GDP growth are slightly larger with the former dataset than with the latter, in particular during the 2007-2009 recession period. While there is substantial uncertainty about responses and contributions, this result might be due to variable omission (reduced conditioning information) when working with the GDP-only dataset. Results on the effects of uncertainty shocks on the U.S. GDP growth are also different in bivariate and multivariate models in Carriero, Clark, and Marcellino (2019).

To put the importance of uncertainty shocks in broader perspective, it may help to compare their effects to those of U.S. monetary policy shocks, which have been extensively studied. While space constraints preclude a detailed analysis in the paper, we have considered estimates of policy shock effects in the literature and in some checks with our own data. These comparisons indicate that some aspects of the effects of uncertainty shocks resemble those of U.S. policy shocks, in both directions of responses and quantitative importance. For example, a surprise increase in uncertainty significantly reduces economic activity and stock prices. The effects of an uncertainty shock differ from the effects of a policy shock in that the former does not consistently produce a fall in prices (however, recall that a fall in prices results from a policy shock at least partly by definition of a policy shock identified by sign restrictions) or foreign interest rates. As measured by variance contributions, in qualitative terms the importance of an international uncertainty shock resembles that of a monetary policy shock: at medium horizons, the contributions are small (for some economic activity measures, the contributions are modest at short horizons). For example, at an 8-quarter ahead horizon, our estimates put the forecast error variance shares of a shock to global uncertainty at about 5 percent for U.S. GDP, less than 3 percent for E.A. and U.K. GDP, and less than 2 percent for stock prices and short-term interest rates. 


\section{Robustness}

In the first of a few robustness checks, we compare our BVAR-GFSV estimates of impulse responses to estimates from a two-step approach similar to those used in a number of uncertainty analyses, such as Jurado, Ludvigson, and Ng (2015) and Berger, Grabert, and Kempa (2016).9 In the first step of the two-step approach, we obtained a measure of uncertainty as the first principal component of log volatilities (posterior medians of $\ln \lambda_{i, t}$ ) estimated with the BVARSV specification. In the second step, we added this uncertainty measure to a homoskedastic BVAR in the 67 variables of the larger dataset - hence yielding a 68-variable BVAR - and performed standard structural analysis, ordering the uncertainty measure first in the system 10

In the supplement, Appendix Figure 2 compares the two-step and BVAR-GFSV estimates. Qualitatively, the impulse responses obtained from the two-step approach are similar to those obtained with our BVAR-GFSV model. In the two-step estimates, as in our BVAR-GFSV results, an international shock to macroeconomic uncertainty gradually dies out over several quarters. The heightened uncertainty reduces GDP and many of its components, including investment, exports, and imports, in the U.S., E.A., and U.K. (although, for the U.K., the responses of exports and imports are smaller in the two-step approach). Other components of spending (e.g., consumption) are reduced in some economies (U.S. and U.K.) but not others (E.A.). In most but not all economies, employment falls and unemployment rises, and some other measures of economic activity, including confidence or sentiment indicators and capacity utilization, also fall. In response, stock prices and policy rates move lower in all three economies (in the BVAR-GFSV estimates, policy rates do not decline uniformly across economies).

While qualitatively similar across the approaches, it is often, although not always, the case that the magnitudes of responses are smaller in the two-step estimates than in the BVAR-GFSV results. This is particularly true in the U.S. estimates, but it also applies to some degree for the E.A. and U.K. For example, in the U.S. results, the declines in GDP, exports, and imports are smaller (in absolute value) in the two-step estimates than in the BVAR-GFSV estimates. In the U.K. results, the decline in GDP is similar across the estimates, but the estimated falloff in exports and imports is not quite as sharp in the two-step estimates as in the baseline estimates.

\footnotetext{
${ }^{9}$ In light of possible distortions of estimated policy responses stemming from the binding lower bound constraint on short-term interest rates following the most recent global recession, we have also considered a robustness check of replacing the policy rates with historical time series of shadow rates. In particular, we have estimated the 3-economy model with the $\mathrm{Wu}$-Xia shadow rate series replacing the short-term interest rates in the baseline model. The resulting estimates of macroeconomic uncertainty and impulse responses to an uncertainty shock are essentially the same as those reported in the paper.

${ }^{10}$ The supplemental appendix's section 3.3 details the priors for this model. Estimates are based on samples of 5,000 retained draws, obtained by sampling a total of 6,000 draws and discarding the first 1,000.
} 
Finally, a key difference is that the confidence bands are wider for the BVAR-GFSV estimates than for the two-step estimates; as might be expected, by treating the uncertainty measure as data rather than an estimate, the two-step approach appears to understate uncertainty around estimates of the effects of shocks to uncertainty.

In results not shown in the interest of brevity, we have also used the two-step approach to consider the effects of a second volatility or uncertainty factor, by adding the first two principal components of BVAR-SV volatilities to a homoskedastic BVAR in the macroeconomic variables, ordering the factors first in the system. These two-step estimates corroborate the difficulty of identifying a second uncertainty factor with effects on the levels of macroeconomic variables. In the two-step case, the shock to the second principal component reduces some selected measures of economic activity in the U.S. but does not have broadly significant effects across economies. In fact, in the U.K. responses, although GDP falls, employment rises and unemployment falls, contradicting most other evidence on the effects of an uncertainty shock, including our preferred BVAR-GFSV estimates presented earlier and the estimates of Jurado, Ludvigson, and Ng (2015) and Carriero, Clark, and Marcellino (2018).

We have also used a two-step approach to provide another check on the degree of spillover in uncertainty from one economy to another. As indicated earlier in the paper, our main results yield a strong commonality in uncertainty rather than a leading role for the U.S. as a driver of global uncertainty. As a further check of spillover, we included in a BVAR country-specific measures of uncertainty obtained by estimating the one-factor BVAR-GFSV model detailed in the paper's section 5.1 separately for the U.S., E.A., and U.K. We produced impulse responses to shocks to these uncertainty measures under different orderings. In these estimates, the countryspecific uncertainty shocks get transmitted to the other economies and have negative effects qualitatively similar to those of the global uncertainty shocks. This pattern is broadly similar to that of Caggiano, Castelnuovo and, Figueres (2019) and Belke and Osowski (2019) for the case of U.S. economic policy uncertainty shocks. These robustness results are consistent with our main findings of strong commonality in uncertainty that affects all major economies considered.

Finally, we used a two-step approach to verify the distinction of the global uncertainty measure from U.S.-specific uncertainty. We specified a BVAR in the 26 U.S. variables of our 3economy dataset, the global uncertainty estimate from the 3-economy BVAR-GFSV model, and the U.S.-specific estimate of uncertainty from Carriero, Clark, and Marcellino (2018), with U.S. uncertainty ordered first, global uncertainty second, and the remaining variables following in the sequence of the baseline model. We then estimated impulse responses for a shock to global uncertainty and obtained results very similar to those in the baseline results above, with a shock 
to global uncertainty significantly depressing a range of measures of economic activity and stock prices, with responses comparable to those in the paper. These results support the baseline specification, in which uncertainty has a global component distinct from U.S.-specific uncertainty. In these estimates, both global and U.S.-specific uncertainty affect the economy.

\section{Conclusions}

This paper uses large Bayesian VARs to measure international macroeconomic uncertainty and its effects, using two datasets, one consisting of GDP growth for 19 industrialized economics and the other comprised of 67 variables in quarterly data for the U.S., E.A., and U.K. Using basic factor model diagnostics, we first provide evidence of significant commonality in international macroeconomic volatility, with one common factor - in each of our datasets - accounting for strong comovement across economies and variables. We then turn to measuring uncertainty and its effects with a large, heteroskedastic VAR in which the error volatilities evolve over time according to a factor structure. The volatility of each variable in the system reflects time-varying common (global) components and idiosyncratic components. In this model, global uncertainty is allowed to contemporaneously affect the macroeconomies of the included nations - both the levels and volatilities of the included variables. In this setup, uncertainty and its effects are estimated in a single step within the same model. Our estimates yield new measures of international macroeconomic uncertainty, and indicate that uncertainty shocks (surprise increases) lower GDP, as well as many of its components, around the world, adversely affect labor market conditions, lower stock prices, and in some economies lead to an easing of monetary policy.

Our analysis extends recent work on common international aspects of macroeconomic uncertainty and its effects in several directions. Our framework allows us to coherently estimate uncertainty and its effects in one step, rather than rely on a two-step approach common in

the uncertainty literature, in which a measure of uncertainty is estimated in a preliminary step and then used as if it were observable data in the subsequent econometric analysis (ignoring time-varying second moments) of its impact on macroeconomic variables. Our approach, unlike some other analyses in the international uncertainty literature, makes use of large datasets; some other work in the U.S.-focused literature has emphasized some benefits to using relatively large cross sections. Finally, whereas some previous work in the international uncertainty literature has either focused on international components to second moments or possibly confounded firstmoment shocks with second-moment changes, our paper cleanly distinguishes uncertainty as a second-moment phenomenon that can affect first moments. 
Our results can be seen as providing an empirical basis for further work on structural openeconomy models. As noted in the introduction, Gourio, Siemer, and Verdelhan (2013) develop a model in which one particular type of uncertainty, associated with disaster risk, leads to a broad decline in economic activity, more so in an economy more affected by the disaster. Mumtaz and Theodoridis (2017) develop a model that can explain international comovement in volatilities. Further work is needed to establish models in which an international shock to risk in the tradition of closed-economy studies such as Bloom (2009), Basu and Bundick (2017), and Leduc and Liu (2016) produces global changes in economic activity and other indicators in line with the patterns documented in this paper.

\section{References}

Ahn, Seung C., and Alex R. Horenstein (2013), "Eigenvalue Ratio Test for the Number of Factors," Econometrica 81, 1203-1227.

Baker, Scott R., Nicholas Bloom, and Steven J. Davis (2016), "Measuring Economic Policy Uncertainty," Quarterly Journal of Economics 131, 1593-1636.

Basu, Susanto, and Brent Bundick (2017), "Uncertainty Shocks in a Model of Effective Demand," Econometrica 85, 937-958.

Belke, Ansgar and Thomas Osowski (2019), "International Effects of Euro Area versus U.S. Policy Uncertainty: A FAVAR Approach," Economic Inquiry 57, 453-481.

Benati, Luca (2016), "Economic Policy Uncertainty, the Great Recession, and the Great Depression,"manuscript, University of Bern.

Berger, Tino, Sibylle Grabert, and Bernd Kempa (2016), "Global and Country-Specific Output Growth Uncertainty and Macroeconomic Performance," Oxford Bulletin of Economics and Statistics 78, 694-716.

Bhattarai, Saroj, Arpita Chatterjee, and Woong Yong Park (2019), "Global Spillover Effects of U.S. Uncertainty," Journal of Monetary Economics, forthcoming.

Bloom, Nicholas (2014), "Fluctuations in Uncertainty," Journal of Economic Perspectives 28, 153-176.

Bloom, Nicholas (2009), "The Impact of Uncertainty Shocks," Econometrica 77, 623-685.

Caggiano, Giovanni, Efrem Castelnuovo, and Juan Manuel Figueres (2017), "Economic Policy Uncertainty and Unemployment in the United States: A Nonlinear Approach," Economics Letters 151, 31-34.

Caggiano, Giovanni, Efrem Castelnuovo, and Juan Manuel Figueres (2019), "Economic Policy 
Uncertainty Spillovers in Booms and Busts," Oxford Bulletin of Economics and Statistics, forthcoming.

Caggiano, Giovanni, Efrem Castelnuovo, and Nicolas Groshenny (2014), "Uncertainty Shocks and Unemployment Dynamics: An Analysis of Post-WWII U.S. Recessions," Journal of Monetary Economics 67, 78-92.

Caggiano, Giovanni, Efrem Castelnuovo, and Giovanni Pellegrino (2017), "Estimating the Real Effects of Uncertainty at the Zero Lower Bound," European Economic Review 100, 257-272. Caldara, Dario, Cristina Fuentes-Albero, Simon Gilchrist, and Egon Zakrajsek (2016), "The Macroeconomic Impact of Financial and Uncertainty Shocks," European Economic Review 88, 185-207.

Carriere-Swallow, Yan, and Luis Felipe Cespedes (2013), "The Impact of Uncertainty Shocks in Emerging Economies," Journal of International Economics 90, 316-325.

Carriero, Andrea, Todd E. Clark, and Massimiliano Marcellino (2018), "Measuring Uncertainty and Its Impact on the Economy," Review of Economics and Statistics 100, 799-815.

Carriero, Andrea, Todd E. Clark, and Massimiliano Marcellino (2019), "The Identifying Information in Vector Autoregressions with Time-Varying Volatilities: An Application to Endogenous Uncertainty," manuscript.

Casarin, Roberto, Claudia Foroni, Massimiliano Marcellino, and Francesco Ravazzolo (2018), "Uncertainty through the Lenses of a Mixed-Frequency Bayesian Panel Markov Switching Model," Annals of Applied Statistics 12, 2559-2586.

Castelnuovo, Efrem, and Trung Duc Tran (2017), "Google it Up! A Google Trends-based Uncertainty Index for the United States and Australia," Economics Letters 161, 149-153.

Cesa-Bianchi, Ambrogio, M. Hashem Pesaran, and Alessandro Rebucci (2019), "Uncertainty and Economic Activity: A Multi-Country Perspective," Review of Financial Studies, forthcoming. Cross, Jamie, Chenghan Hou, and Aubrey Poon (2018), "International Transmission of Macroeconomic Uncertainty in Small Open Economies: An Empirical Approach," CAMP WP No 12/2018, Norwegian Business School.

Cross, Jamie, Timothy Kam, and Aubrey Poon (2018), "Uncertainty Shocks in Markets and Policies: What Matters for a Small Open Economy?" manuscript.

Cuaresma, Jesus Crespo, Florian Huber, and Luca Onorante (2017), "The Macroeconomic Effects of International Uncertainty Shocks," Working Paper No. 245, Vienna University of Economics and Business.

Davis, Steven J. (2016), "An Index of Global Economic Policy Uncertainty," NBER Working Paper No. 22740. 
Fernandez-Villaverde, Jesus, Pablo Guerron-Quintana, Juan F. Rubio-Ramirez, and Martin Uribe (2011), "Risk Matters: The Real Effects of Volatility Shocks," American Economic Review 101, 2530-2561.

Gilchrist, Simon, Jae W. Sim, and Egon Zakrajsek (2014), "Uncertainty, Financial Frictions, and Investment Dynamics," NBER Working Paper no. 20038.

Gourio, Francois, Michael Siemer, and Adrien Verdelhan (2013), "International Risk Cycles," Journal of International Economics 89, 471-484.

Jarocinski, Marek, and Bartosz Mackowiak (2017), "Granger Causal Priority and Choice of Variables in Vector Autoregressions," Review of Economics and Statistics 99, 319-329.

Jurado, Kyle, Sydney C. Ludvigson, and Serena Ng (2015), "Measuring Uncertainty," American Economic Review 105, 1177-1216.

Klossner, Stefan, and Rodrigo Sekkel (2014), "International Spillovers of Policy Uncertainty," Economics Letters 124, 508-512.

Leduc, Sylvain, and Zheng Liu (2016), "Uncertainty Shocks Are Aggregate Demand Shocks," Journal of Monetary Economics 82, 20-35.

Ludvigson, Sydney C., Sai Ma, and Serena Ng (2019), "Uncertainty and Business Cycles: Exogenous Impulse or Endogenous Response?" NBER Working Paper 21803, January.

Mumtaz, Haroon, and Konstantinos Theodoridis (2017), "Common and Country Specific Economic Uncertainty," Journal of International Economics 105, 205-216.

OECD (2017), Quarterly GDP (indicator).

Ramey, Garey and Valerie A. Ramey (1995) "Evidence on the Link Between Volatility and Growth," American Economic Review 85, 1138-1151.

Rossi, Barbara, and Tatevik Sekhposyan (2017), "Macroeconomic Uncertainty Indices for the Euro Area and its Individual Member Countries," Empirical Economics 53, 41-62.

Rossi, Barbara, Tatevik Sekhposyan, and Matthieu Soupre (2018), "Understanding the Sources of Macroeconomic Uncertainty," manuscript.

Stock, James H., and Mark W. Watson (2016), "Factor Models for Macroeconomics" (pp. 415525), in John B. Taylor and Harald Uhlig, eds., Handbook of Macroeconomics, Vol. 2 (Amsterdam: North Holland).

Stock, James H., and Mark W. Watson (2012), "Disentangling the Channels of the 2007-2009 Recession," Brookings Papers on Economic Activity (2012), Spring, 81-130. 
Table 1: Factor loadings: 19-country GDP dataset

\begin{tabular}{lcc}
\hline \hline Country & $\begin{array}{c}\text { Principal component } \\
\text { loading }\end{array}$ & $\begin{array}{c}\text { GFSV loading } \\
\text { posterior mean (st. dev.). }\end{array}$ \\
\hline U.S. & 0.939 & $0.925(0.347)$ \\
Australia & 1.051 & $0.695(0.383)$ \\
Austria & 1.093 & $1.060(0.374)$ \\
Belgium & 0.978 & $1.390(0.395)$ \\
Canada & 1.103 & $1.004(0.392)$ \\
Denmark & 0.751 & $0.505(0.446)$ \\
Finland & 1.062 & $1.007(0.333)$ \\
France & 1.079 & $0.718(0.399)$ \\
Germany & 1.105 & $1.634(0.362)$ \\
Italy & 1.106 & $1.169(0.371)$ \\
Japan & 1.065 & $0.915(0.408)$ \\
Luxembourg & 0.939 & $0.985(0.362)$ \\
Netherlands & 0.889 & $0.966(0.396)$ \\
Norway & 0.780 & $0.515(0.395)$ \\
Portugal & 1.003 & $1.287(0.401)$ \\
Spain & 0.943 & $1.415(0.382)$ \\
Sweden & 1.114 & $1.065(0.382)$ \\
Switzerland & 0.769 & $0.396(0.388)$ \\
U.K. & 1.097 & $1.130(0.413)$ \\
\hline \hline
\end{tabular}

Note: The second column provides loadings on a first common factor estimated as the principal component of log volatilities of a BVAR-SV model. The third column provides estimates of the loadings $\beta_{m, i}$ of equation (2) of the one-factor BVAR-GFSV model. 


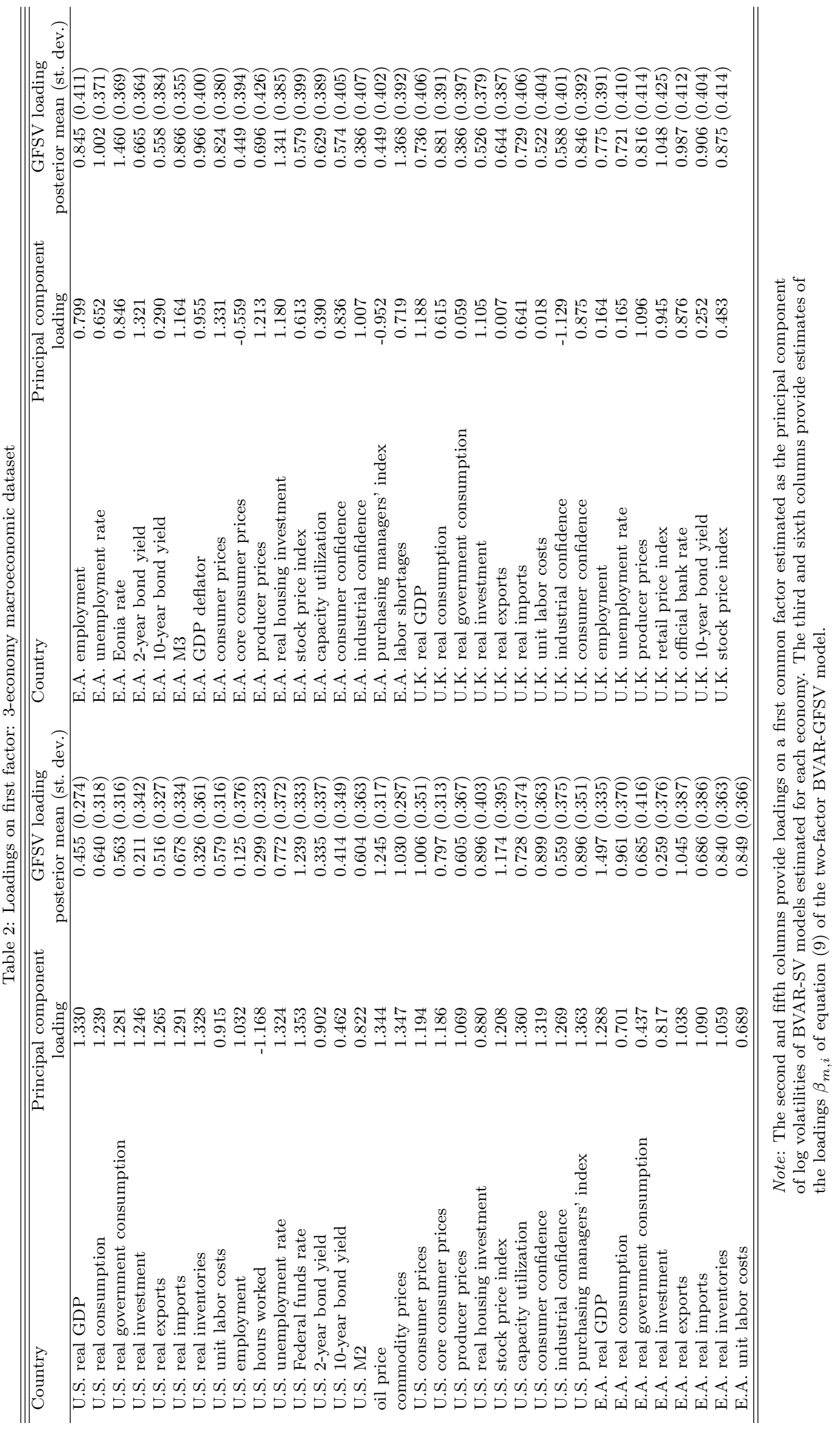



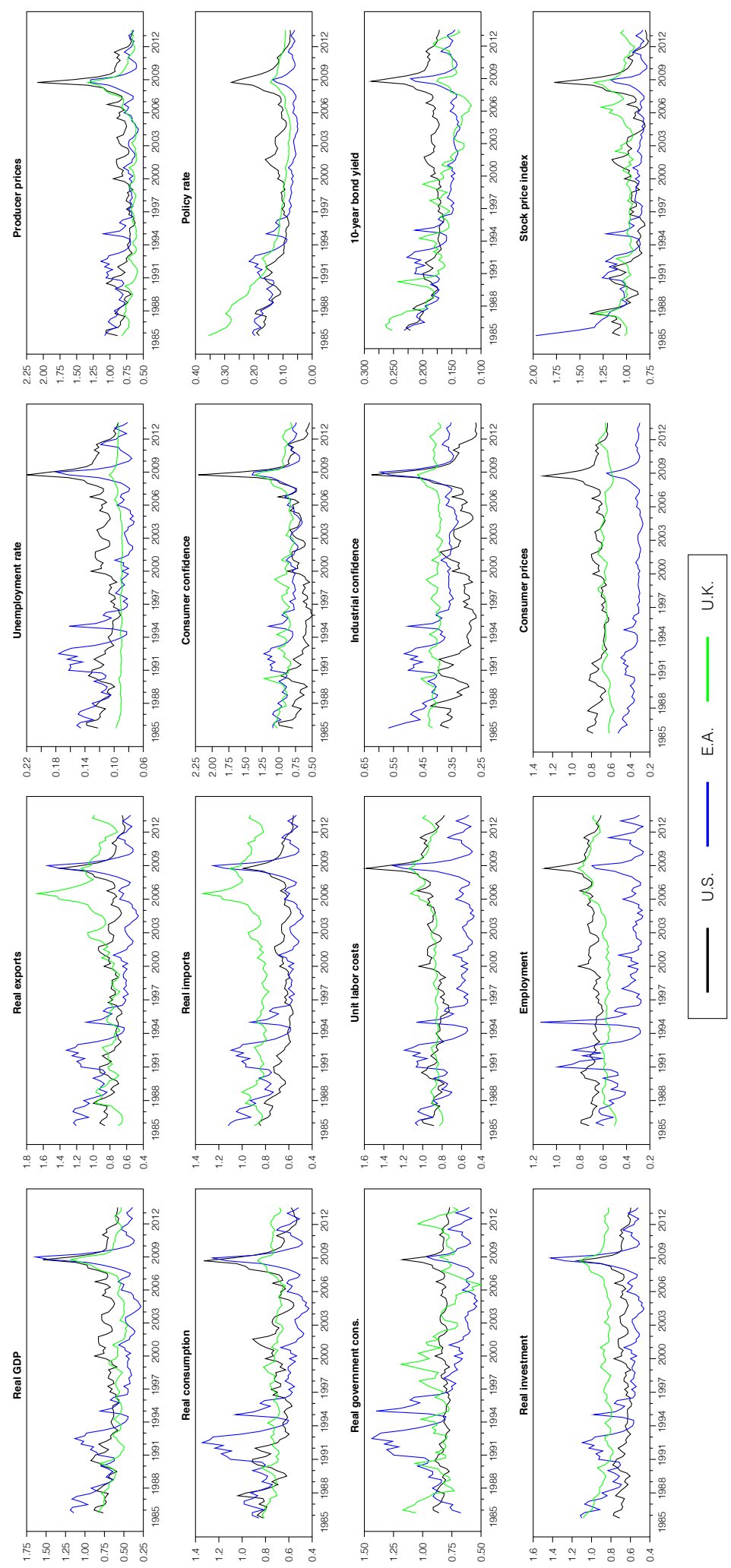

Figure 1: BVAR-SV estimates of volatilities, selected variables. The reported entries are posterior medians of standard deviations of reduced-form innovations from BVAR-SV models estimated for each economy. 

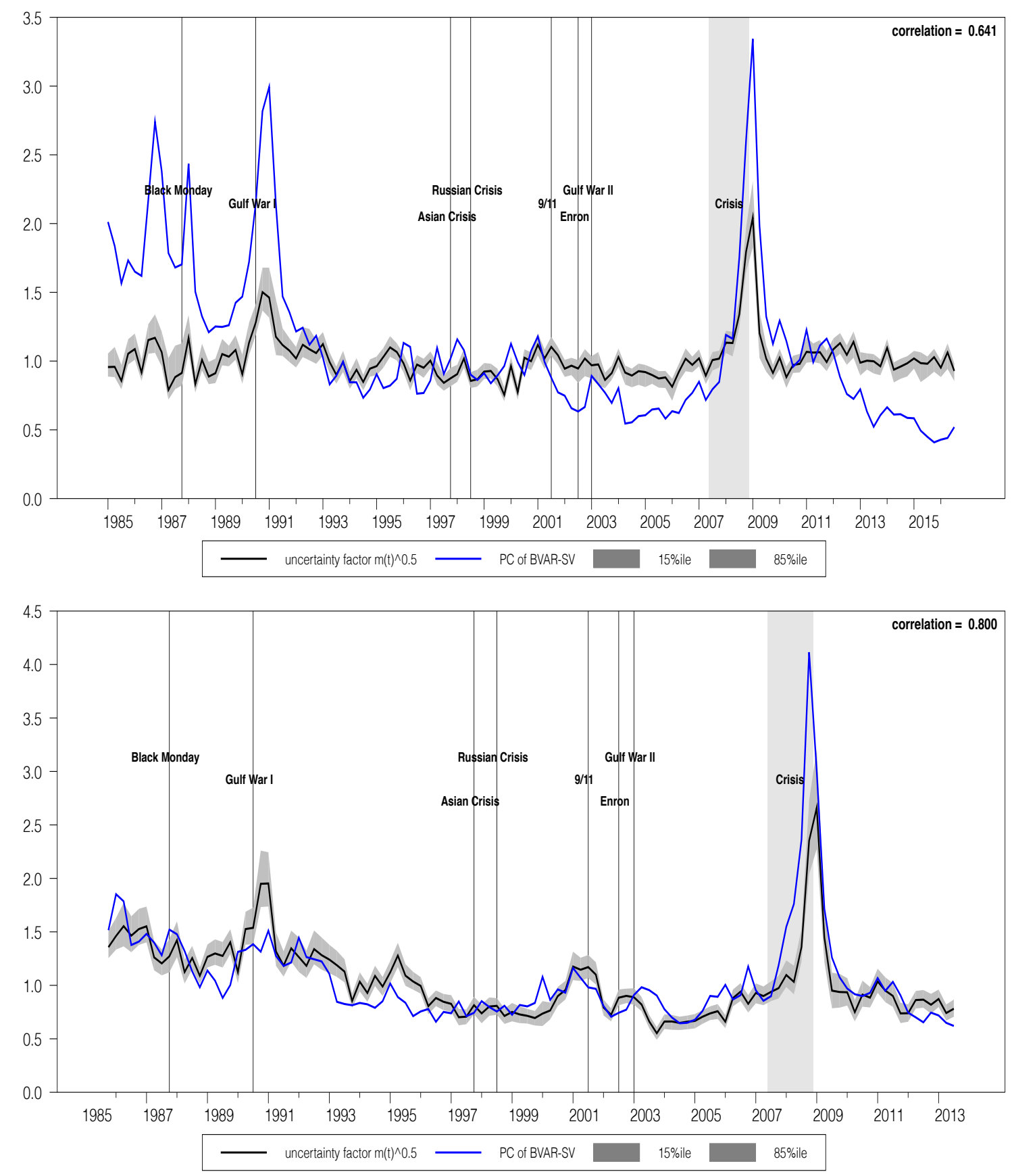

Figure 2: Uncertainty estimates for 19-country GDP dataset in the top panel and for 3-economy macroeconomic dataset in the bottom panel. In each panel, the blue line provides an estimate obtained from the first principal component of the BVAR-SV estimates of log volatility. The solid black line and gray-shaded regions provide the posterior median and $15 \% / 85 \%$ quantiles of the BVAR-GFSV estimate of macroeconomic uncertainty $\left(m_{t}^{0.5}\right)$. The periods indicated by black vertical lines or regions correspond to the uncertainty events highlighted in Bloom (2009). Labels for these events are indicated in text horizontally centered on the event's start date. 

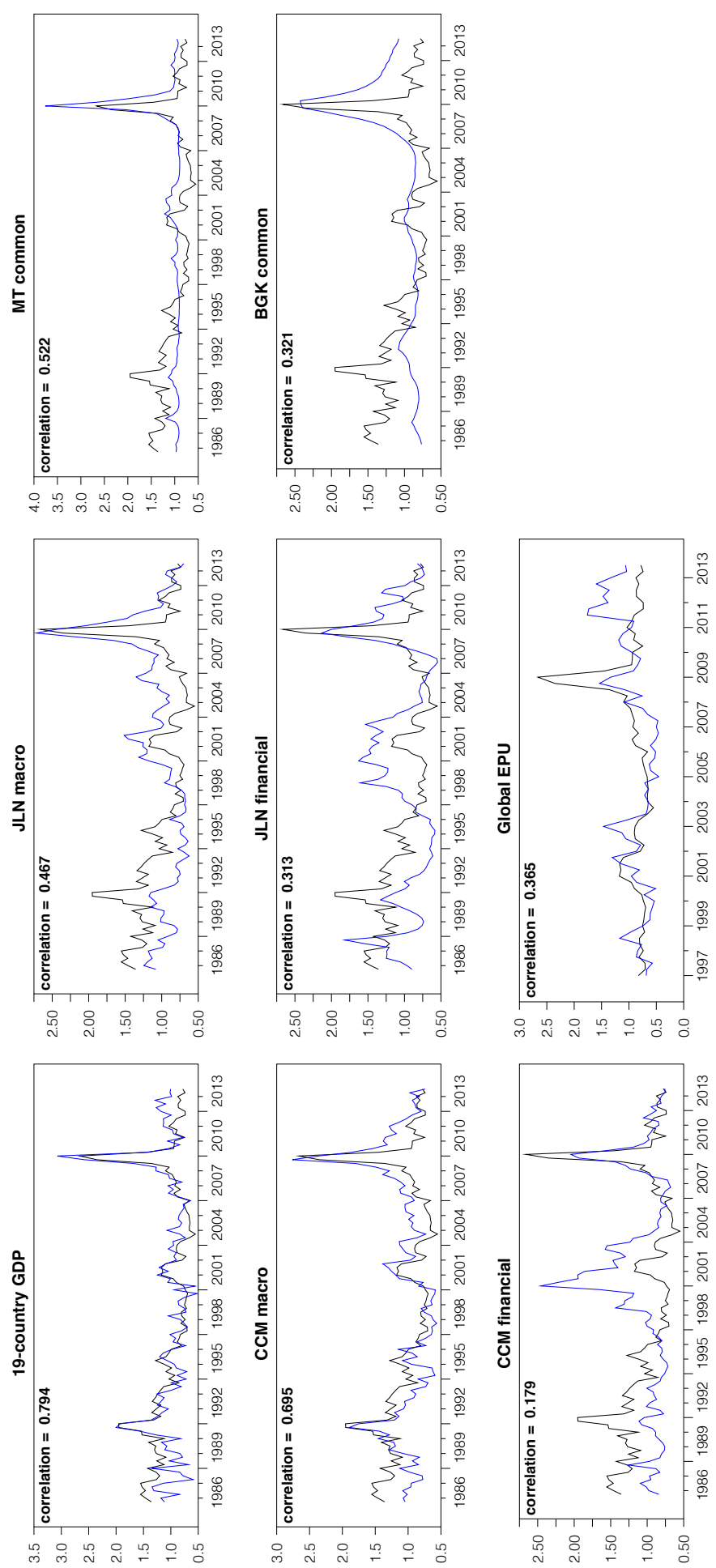

Figure 3: Comparison of uncertainty estimates to others in the literature. The top left panel compares the uncertainty estimate obtained from the 3-economy macroeconomic dataset (black line) to that obtained with the 19-country GDP dataset (blue line). Other panels compare the 3-economy macroeconomic dataset estimate (black line) to a different estimate (blue line) from the literature, normalized to have the same mean and variance as the 3-economy macroeconomic dataset estimate. 


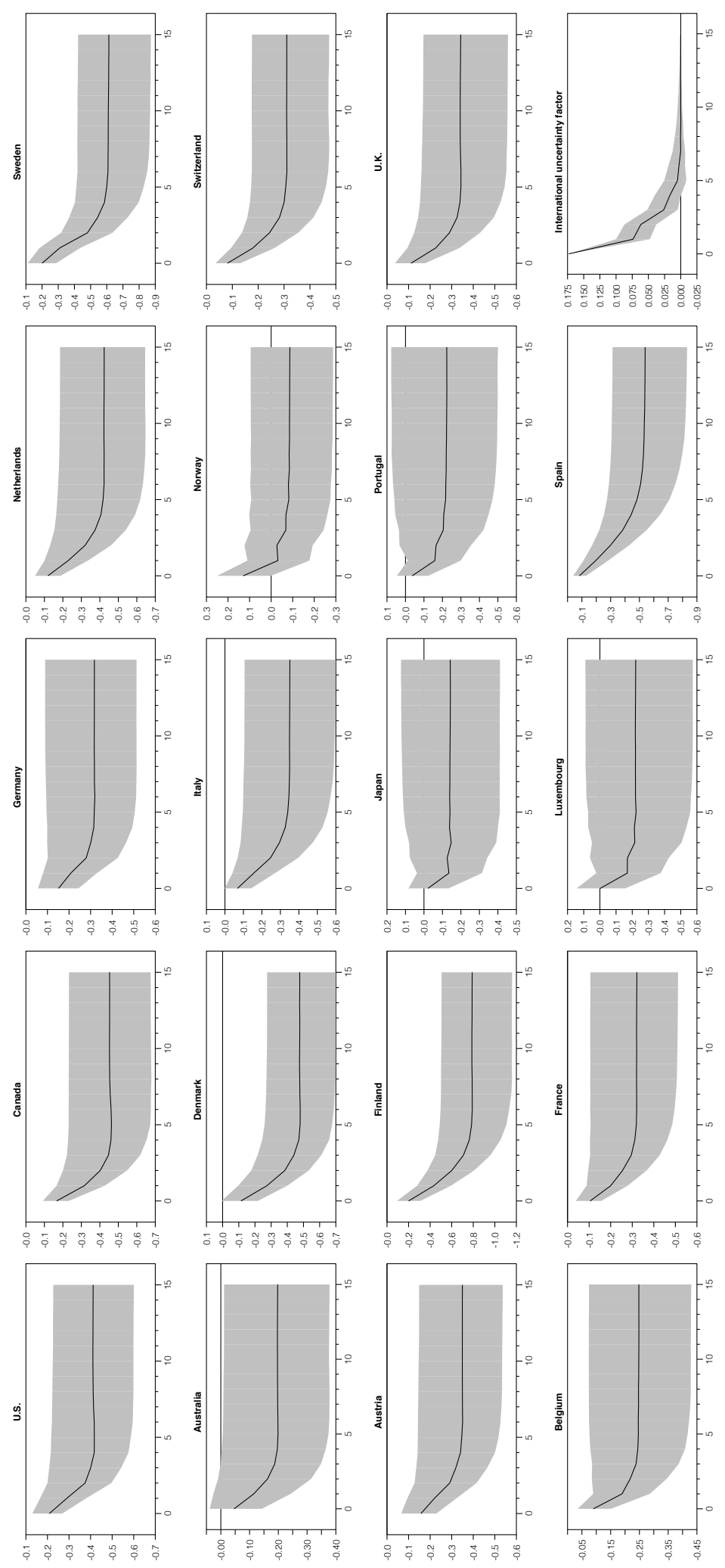

Figure 4: Impulse responses for international uncertainty shock: one-factor BVAR-GFSV estimates for 19-country GDP dataset, posterior median (black line) and 15\%/85\% quantiles 

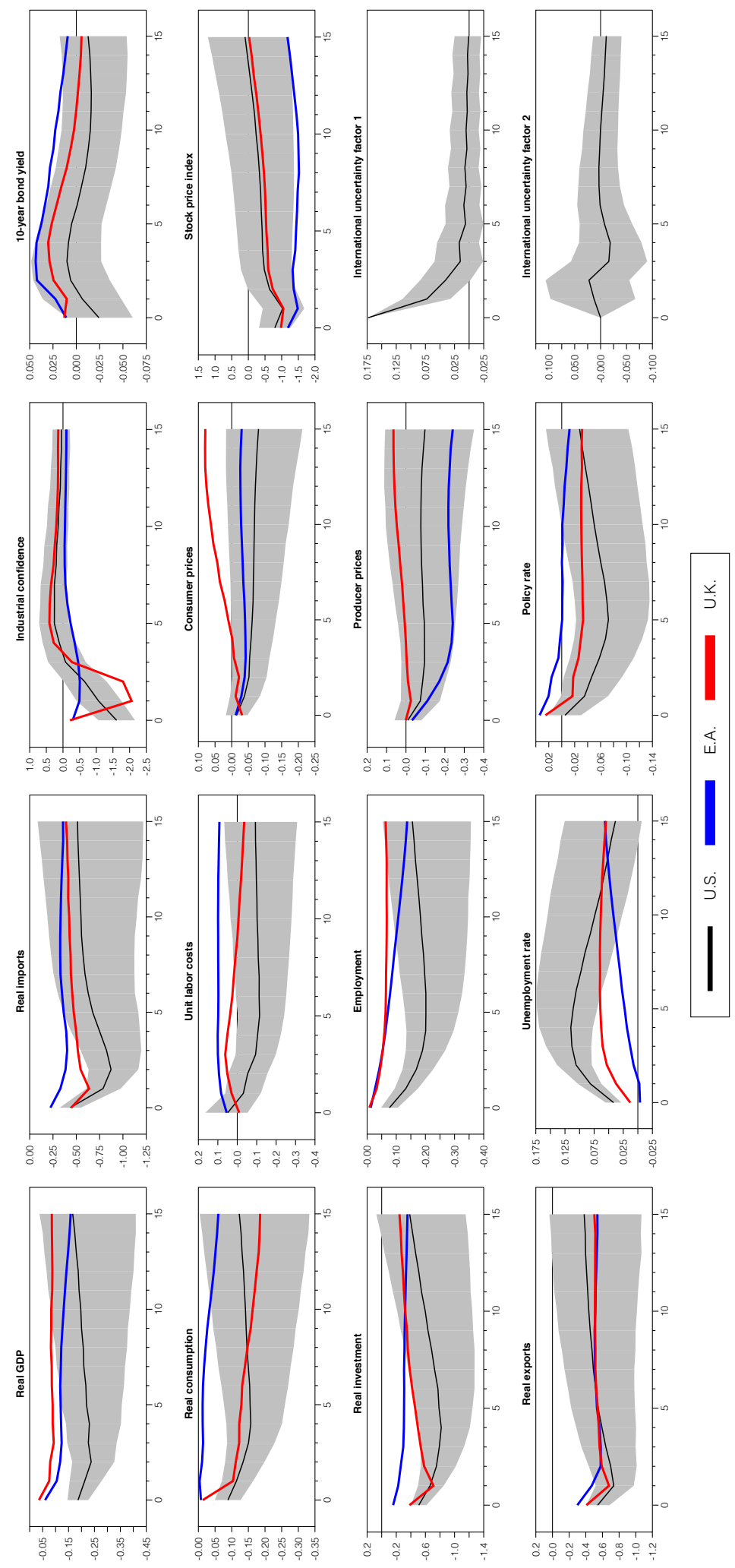

Figure 5: Impulse responses for international uncertainty shock: two-factor BVAR-GFSV estimates for 3-economy macroeconomic dataset, selected variables. The black line and gray shading provide posterior medians and 15\%/85\% quantiles for the U.S. response. The colored lines provide posterior medians for the E.A. and U.K. 

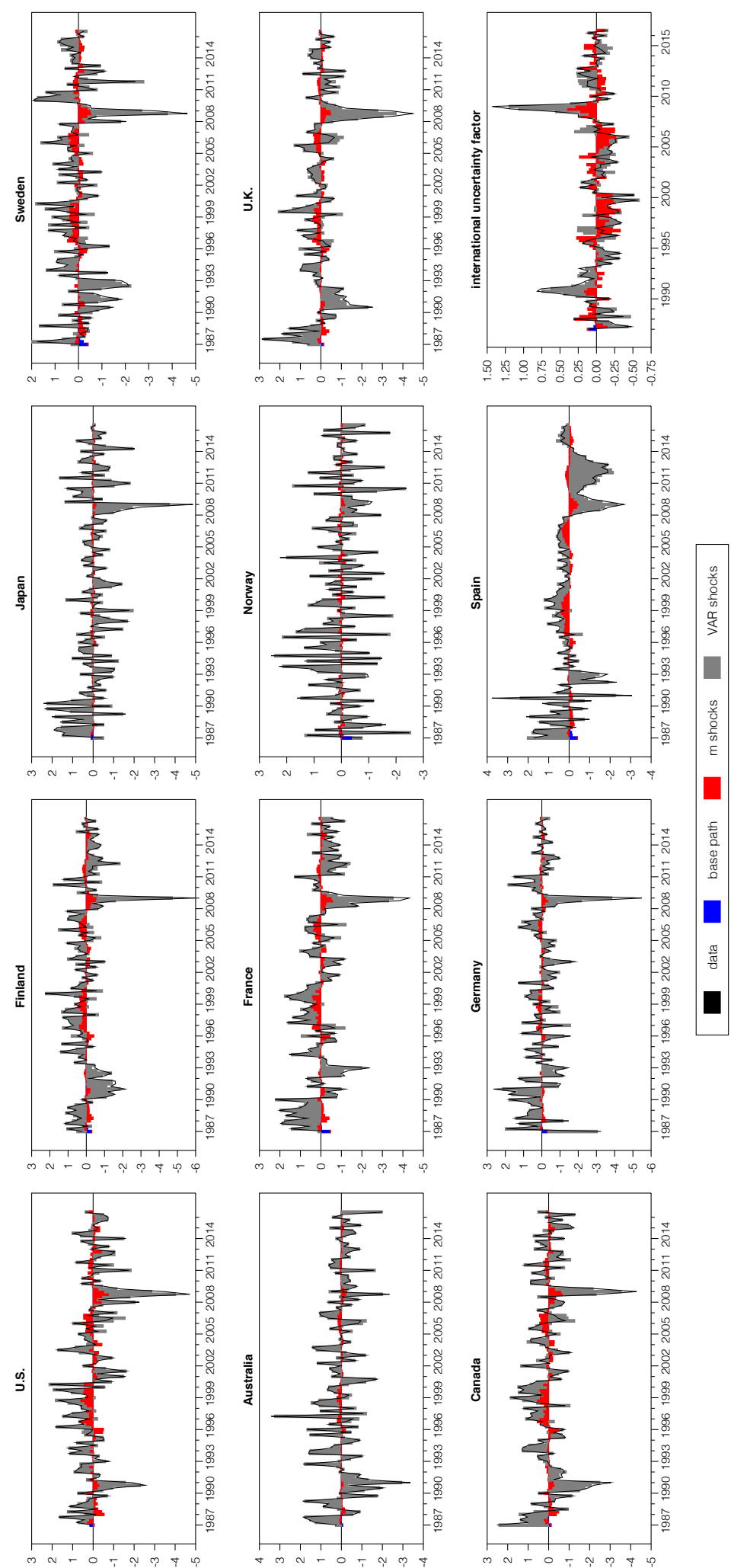

Figure 6: Historical decompositions: one-factor BVAR-GFSV estimates for 19-country GDP dataset, selected variables, posterior medians 

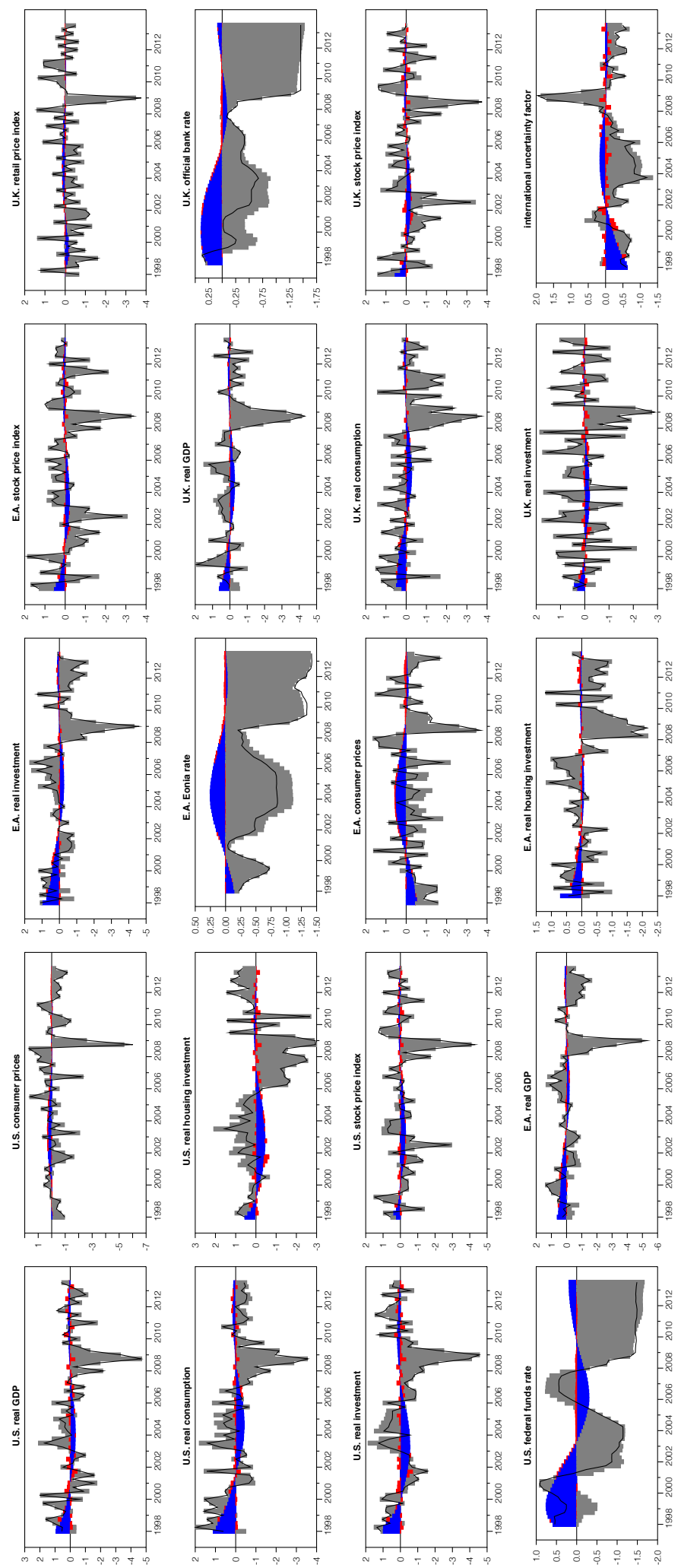

Figure 7: Historical decompositions: two-factor BVAR-GFSV estimates for 3-economy macroeconomic dataset, selected variables, posterior medians 